\title{
Vancomycin and linear IgA bullous dermatosis
}

\author{
Amr Ismail MD, Rocio Gavidia Quezada MD, Kenneth Iwuji MD, Abdussalam Shredi MD, Michelle Tarbox MD
}

\begin{abstract}
Vancomycin is a broad-spectrum antibiotic that is considered an optimal parenteral treatment for many infections, including septicemia, pneumonia, cellulitis, endocarditis, and meningitis caused by methicillin-resistant Staphylococcus aureus. It is the first-line treatment for the increasing health care-associated infection Clostridium difficile pseudomembranous colitis. More side effects are being reported secondary to increased vancomycin use. Of those side effects, skin reactions are becoming more recognized by physicians.
\end{abstract}

Keywords: Vancomycin, methicillin-resistant Staphylococcus aureus, linear IgA bullous dermatosis, vancomycin skin reactions

\section{INTRODUCTION}

In the past few years, beta-lactam-resistant Gram-positive bacteria, including methicillin-resistant Staphylococcus aureus have become prevalent nosocomial pathogens in the United States. Vancomycin hydrochloride has been the most effective and reliable drug for treating methicillin-resistant Staphylococcus aureus. This has increased vancomycin usage considerably. ${ }^{1,2}$

Increased vancomycin use has led to increased reports of adverse events, and skin reactions are the most frequent reason for discontinuation of the medication. ${ }^{2}$ These include linear IgA bullous dermatosis (LABD), drug rash eosinophilia, and Stevens-Johnson syndrome/toxic epidermal necrolysis. ${ }^{3}$ Linear IgA bullous dermatosis is a rare, idiopathic, or drug-induced autoimmune blistering disease characterized by the linear deposition of IgA at the dermo-epidermal junction. The underlying pathophysiologic mechanism that triggers the autoimmune response in LABD still

Corresponding author: Amr Ismail Contact Information: Amr.ismail@ttuhsc.edu DOI: 10.12746/swrccc.v6i22.445 remains largely unknown. Both humoral and cellular immune responses may be involved in the pathogenesis of this disease. Tissue injury resulting from an antibody-induced local inflammatory response and the release of proteolytic enzymes by neutrophils and other inflammatory cells may contribute to the development of skin and mucosal lesions. ${ }^{4}$

\section{CASE}

A 56-year-old man presented to the emergency center complaining of a generalized skin rash with blisters. He has a past medical history of alcoholic cirrhosis with pancytopenia, chronic pancreatitis, and chronic osteomyelitis. Prior to this presentation, he was admitted for osteomyelitis of his left arm and had hardware removed from his arm. The culture of the hardware grew methicillin-resistant Staphylococcus aureus and Pseudomonas aeruginosa. He was discharged three days prior to this presentation on intravenous vancomycin, oral ciprofloxacin for osteomyelitis, and on oral metronidazole for colitis. Initially, he developed a purpuric rash which was itchy and started on his trunk (Figure 1). Over the next three days, the rash progressed rapidly to involve his extremities, back, buttocks, soles, palms, mouth, lips, and tongue. It progressed to hemorrhagic bullae, 


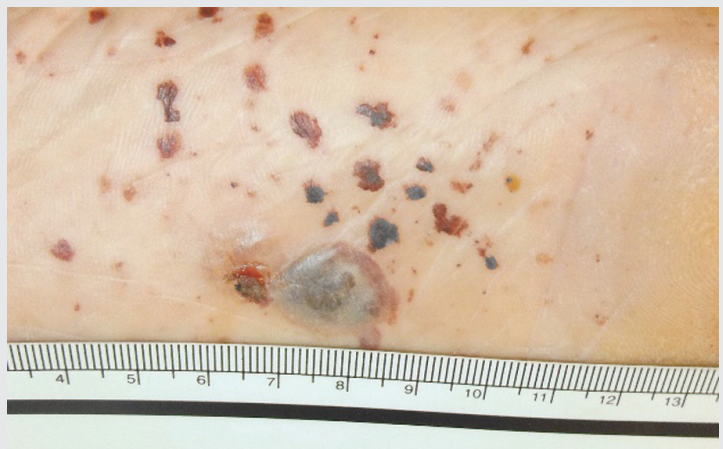

ह

Figure 1. Purpuric rash and bullae on plantar aspect of foot.

most prominent on the back (Figure 2). He denied fever, chills, chest pain, arthralgia, and diarrhea. On admission to our hospital his laboratory values were notable for a platelet count of $6000 / \mu \mathrm{L}$ for which he was transfused two units of platelets with no significant improvement. Vancomycin was not restarted on admission. Dermatology was consulted, and a skin biopsy was done. The biopsy showed sub-epidermal

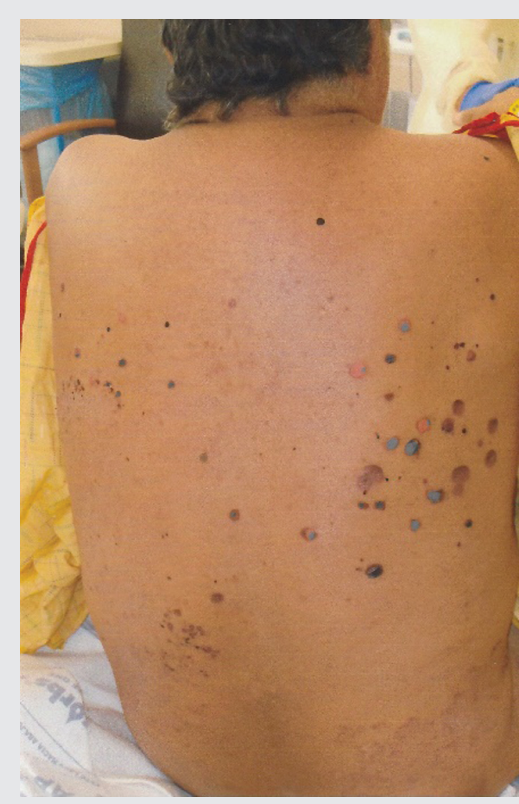

Figure 2. Purpuric rash and bullae in various stages on back.

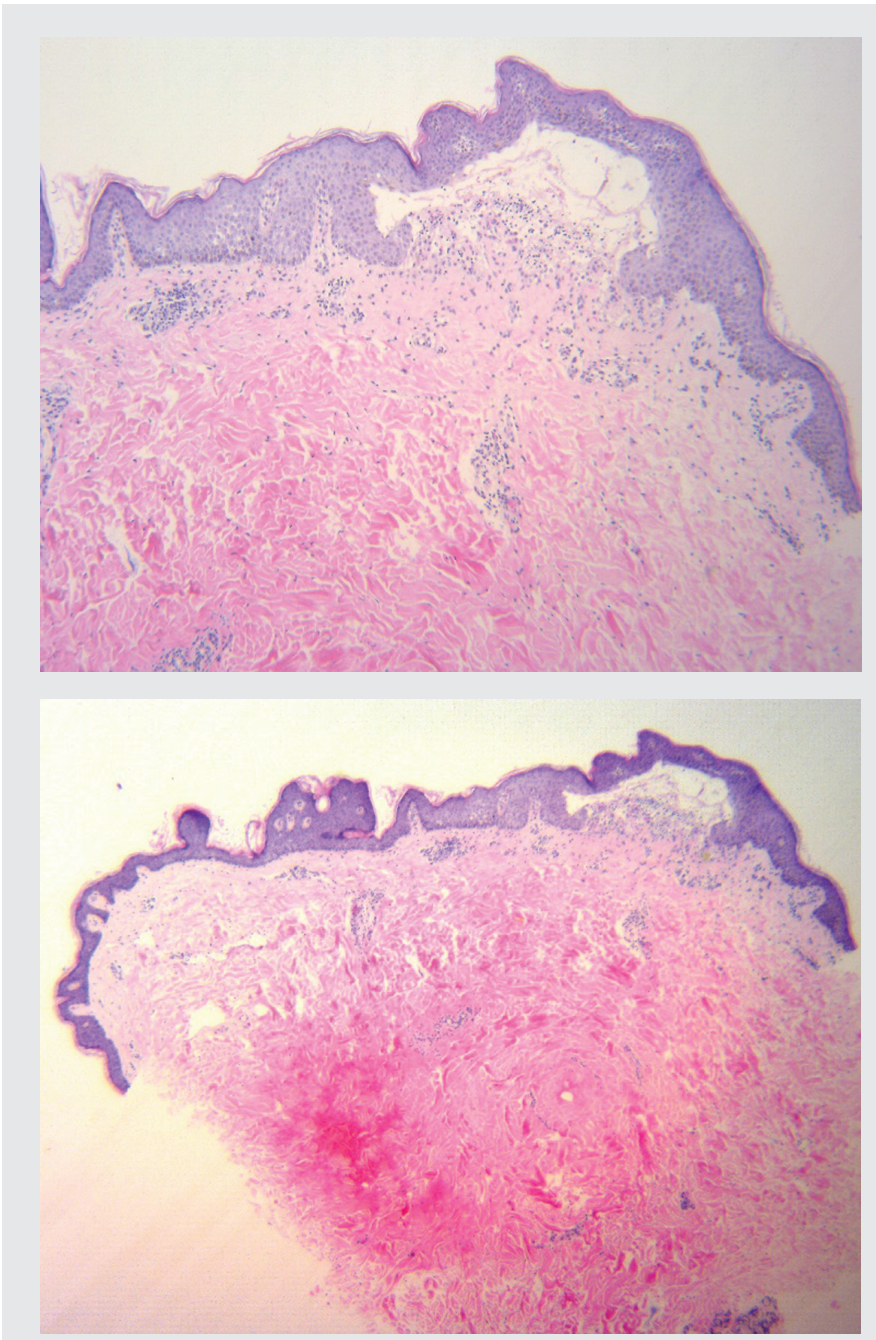

Figure 3. Low power image demonstrating subepidermal blister formation.

blister with neutrophils and eosinophils (Figures 3 and 4). Immunofluorescence showed linear $\lg A$ and $\lg G$ deposition at the dermal/epidermal junction (Figure 5). Dermatology recommended supportive treatment.

The patient was found to have acute-on-chronic thrombocytopenia that did not respond to transfusions. Hematology was consulted, and he was treated for immune thrombocytopenic purpura with one dose of $85 \mathrm{~g}$ of intravenous immunoglobulin and $100 \mathrm{mg}$ of prednisone. This was followed with three doses of 

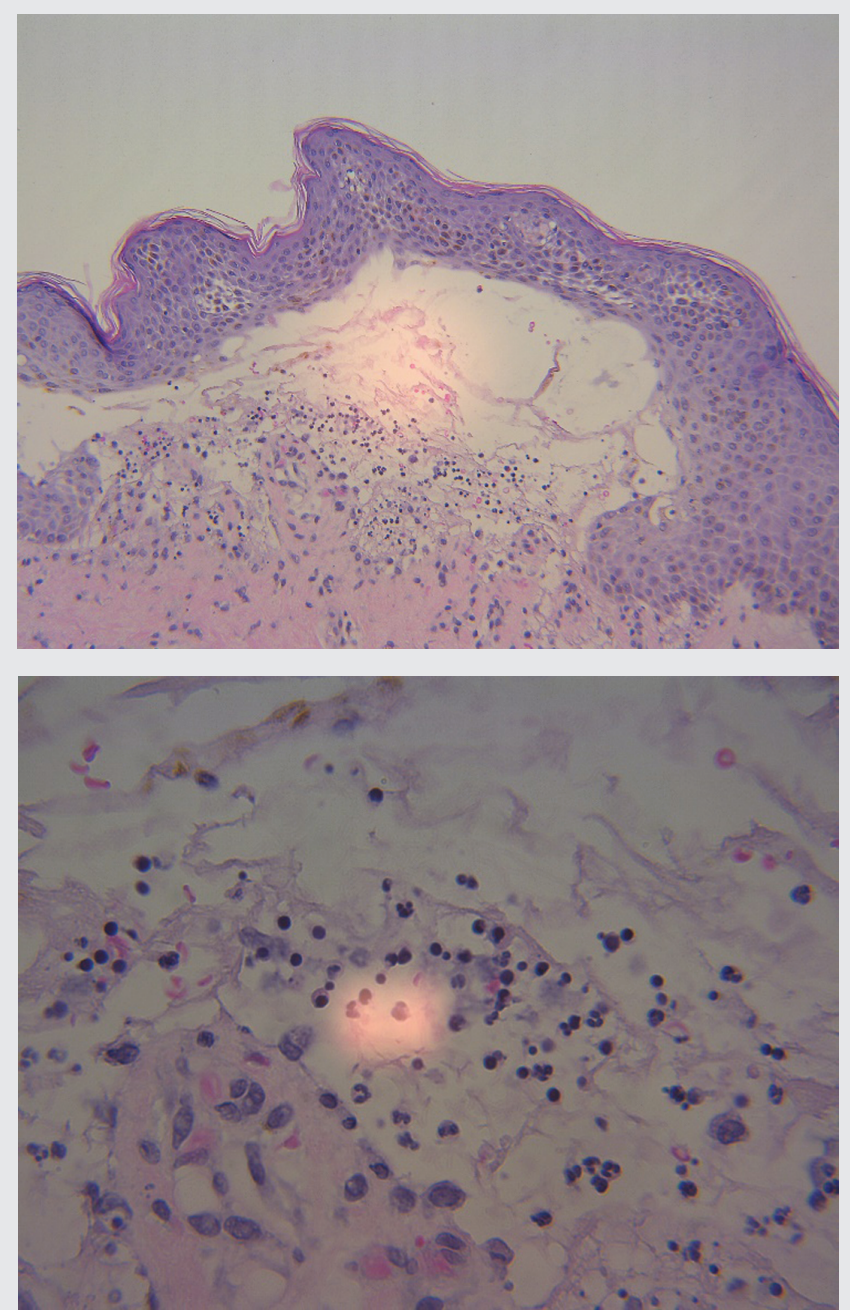

Figure 4. Higher power image demonstrating subepidermal blister cavity with numerous neutrophils.

$40 \mathrm{mg}$ of prednisone over the next 3 days. His platelet count improved to his baseline after one unit of platelets. The patient was hospitalized for a week, and his skin lesions continued to improve daily. It is unclear whether the IVIG and prednisone altered the clinical course of his skin disease. He was discharged on oral ciprofloxacin and was re-challenged with oral metronidazole later with no significant side effects noted. $\mathrm{He}$ was re-admitted for infection at our hospital two months later, and at that time his rash and bullae had completely resolved.

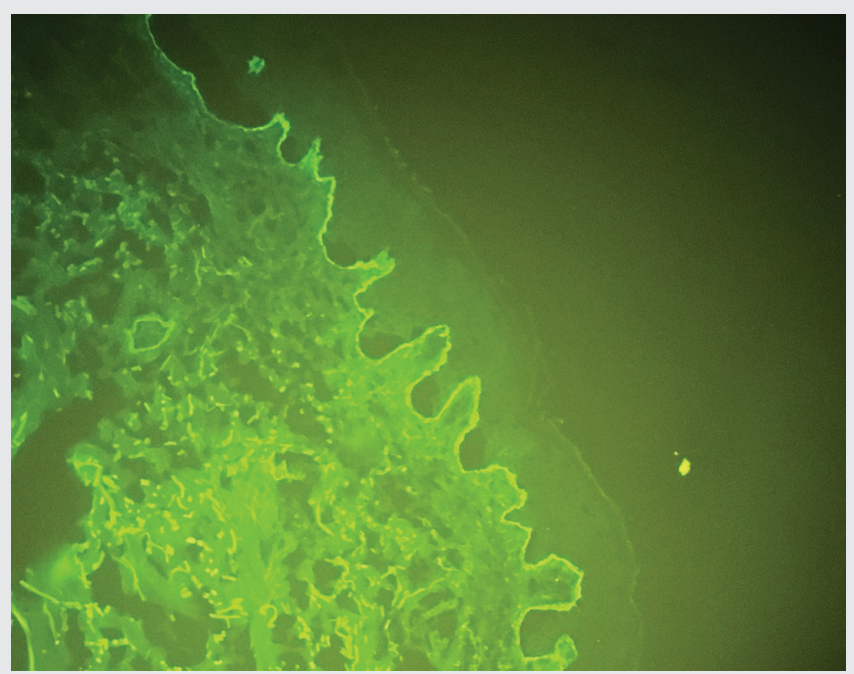

Figure 5. Linear deposits of $\operatorname{IgA}$ at the dermalepidermal basement membrane on direct immunofluorescence.

\section{Discussion}

We report a patient who presented with linear $\lg A$ bullous dermatosis associated with treatment with vancomycin. Linear IgA bullous dermatosis was the most commonly identified vancomycin skin reaction with an incidence ranging from 0.2 to 2.3 cases per million individuals per year usually in patients older than $60 .^{5}$ In one study, LABD made up $48 \%$ of all reported skin reactions with a median onset time of seven days. Linear IgA bullous dermatosis is a sub-epidermal bullous disease defined by the presence of homogeneous linear deposits of IgA at the dermal-epidermal basement membrane on direct immunofluorescence. Histologically, LABD is characterized by sub-epidermal bullae with a predominantly neutrophilic infiltrate and basal cell vacuolization (Figures 1 and 2 ). ${ }^{3}$ Drugs known to induce LABD include vancomycin, NSAIDS, lithium, captopril, amiodarone, phenytoin, cyclosporine, furosemide, and somatostatin. Patients with drug-induced LABD have spontaneous resolution of skin lesions within 1 to 3 weeks after removal of offending drug, and usually no treatment is necessary. Corticosteroids and dapsone do not appear to influence the prognosis. Patients will need supportive care that includes pain 
control, fluid replacement, avoidance of any adhesive material, fluid and electrolyte management, nutritional support, and protection against infection and sepsis. The prognosis of patients with vancomycin induced LABD is excellent. After the vancomycin is discontinued, the patient should have prompt improvement without residual skin lesions. If patients are re-challenged with vancomycin after having vancomycin-induced $\mathrm{LABD}$, they may have a more severe recurrence with a shorter latency and a longer course. ${ }^{3}$ The distribution and the shape of vesicles can help differentiate LABD from other common causes of cutaneous blisters. Usually the bullae are tense differentiating LABD from Stevens-Johnson syndrome (SJS) and toxic epidermal necrolysis (TEN) in which the bullae are flaccid with skin sloughing. The patient usually lacks signs of systemic illness that occur with SJS/TEN. Skin biopsy will confirm the diagnosis of LABD.

According to a Scandinavian retrospective study that evaluated 23 patients with histologic diagnosis of $\angle A B D$, the initial working diagnosis in these patients included bullous pemphigoid, dermatitis herpetiformis, Steven-Johnson syndrome, bullous dermatitis, erythema multiforme, and others. ${ }^{6}$ Therefore, it is important to consider the clinical findings as well as immune and histologic studies to confirm the diagnosis, as treatment can vary significantly if LABD is confirmed. Kidney dysfunction is the main side effect that clinicians monitor in patients treated with vancomycin. However, this case highlights the importance of reporting additional adverse effects from this drug.
Article citation: Ismail A, Gavidia Quezada R, Iwuji K, Shredi A, Tarbox M. Vancomycin and linear IgA bullous dermatosis. Southwest Respiratory and Critical Care Chronicles 2018;6(22):38-41

From: Departments of Internal Medicine (AI, RGQ, KI, AS) and Dermatology (MT) at Texas Tech University Health Sciences Center, Lubbock, TX

Submitted: $10 / 22 / 17$

Accepted: $12 / 22 / 17$

Reviewers: Ashley Sturgeon MD, David Sotello MD Conflicts of interest: none

\section{REFERENCES}

1. VISA/VRSA-Vancomycin-Intermediate/Resistant Staphylococcus aureus fact sheet. Available at: http://www.cdc.gov/ ncidod/hip/ARESIST/visa.htm.

2. Korman TM, Turnidge JD, Grayson ML. Risk factors for adverse cutaneous reactions associated with intravenous vancomycin. J Antimicrob Chemother 1997;39:315-318.

3. Jones DH. Early diagnosis is key in vancomycin-induced linear IgA bullous dermatosis and Stevens-Johnson syndrome. J Am Osteopath Assoc 2004 Apr; 104(4):157-63.

4. Fortuna G, Marinkovich MP. Linear immunoglobulin A bullous dermatosis. Clin Dermatol 2012;30:38.

5. Minhas JS, Immune-mediated reactions to vancomycin: A systematic case review and analysis. Ann Allergy Asthma Immunol 2016 Jun 4; 116(6):544-53.

6. Lings K, Bygum A. Linear IgA bullous dermatosis: a retrospective study of 23 patients in Denmark. Acta Derm Venereol 2015;95:466-471. 